\title{
Article
}

\section{On a nonlinear differential equation with two-point nonlocal condition with parameters}

\author{
A. M. A. El-Sayed ${ }^{1, *}$, M. SH. Mohamed ${ }^{1}$ and E. M. Al-Barg ${ }^{2}$ \\ 1 Faculty of Science, Alexandria University, Alexandria, Egypt. \\ 2 Faculty of Science, Sirt University, Libya. \\ * Correspondence: eman.albarg@gmail.com
}

Received: 2 March 2020; Accepted: 7 July 2020; Published: 31 August 2020.

\begin{abstract}
Here we study the existence of solutions of a nonlocal two-point, with parameters, boundary value problem of a first order nonlinear differential equation. The maximal and minimal solutions will be proved. The continuous dependence of the unique solution on the parameters of the nonlocal condition will be proved. The anti-periodic boundary value problem will be considered as an application.
\end{abstract}

Keywords: Euler-Bernoulli viscoelastic equation, global existence, asymptotic behavior, memory.

MSC: 35G16, 74Dxx, 35B40.

\section{Introduction}

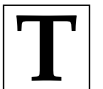

he existence of solutions of the two-point boundary value problem of the first order differential equation

$$
\frac{d x}{d t}=f(t, x(t)), \quad t \in(0, T)
$$

have been studied in [1-3]. Also, some nonlocal problems of differential equations have been considered in [4-17]. Consider the nonlocal two-point boundary value problem with parameters $\alpha$ and $\beta$;

$$
\begin{gathered}
\frac{d x}{d t}=f(t, x(t)), \quad \text { a.e, } \quad t \in(0, T), \\
\alpha x(\tau)+\beta x(\eta)=x_{0}, \quad \tau \in[0, T), \quad \eta \in(0, T], \quad \alpha+\beta \neq 0 .
\end{gathered}
$$

Here we study the existence of at least one absolutely continuous solution $x \in A C[0, T]$ of the Problem (1)-(2). The maximal and minimal solutions of the Problem (1)-(2) will be studied. Also the continuous dependence of the unique solution $x \in A C[0, T]$ on the parameters $\alpha, \beta$ and $x_{0}$ will be proved. The anti-periodic boundary value problem will be considered as an application.

\section{Existence of solutions}

Consider the Problem (1)-(2) under the following assumptions;

(i) $f:[0 . T] \times R \rightarrow R$ is measurable in $t \in[0, T]$ for every $x \in R$ and continuous in $x \in R$ for every $t \in[0, T]$.

(ii) There exist an integrable function $m \in L^{1}[0, T]$ and a constant $b \geq 0$ such that

$$
|f(t, x)| \leq m(t)+b|x| .
$$

(iii) $2 b T<1$.

\subsection{Integral equation representation}

Here we give the integral representation of the solution of the Problem (1)-(2) if it exists. We have the following lemma. 
Lemma 1. If the solution of the Problem (1)-(2) exists, then it can be expressed by the integral equation

$$
x(t)=\frac{1}{\alpha+\beta}\left[x_{0}-\alpha \int_{0}^{\tau} f(s, x(s)) d s-\beta \int_{0}^{\eta} f(s, x(s)) d s\right]+\int_{0}^{t} f(s, x(s)) d s .
$$

Proof. Let the boundary value Problem (1)-(2). Integrating Equation (1), we obtain

$$
x(t)=x(0)+\int_{0}^{t} f(s, x(s)) d s .
$$

For $t=\tau$, we obtain

$$
x(\tau)=x(0)+\int_{0}^{\tau} f(s, x(s)) d s
$$

and

$$
\alpha x(\tau)=\alpha x(0)+\alpha \int_{0}^{\tau} f(s, x(s)) d s
$$

For $t=\eta$ in (4), we obtain

$$
x(\eta)=x(0)+\int_{0}^{\eta} f(s, x(s)) d s
$$

and

$$
\beta x(\eta)=\beta x(0)+\beta \int_{0}^{\eta} f(s, x(s)) d s .
$$

Substituting (5) and (6) into (2), we obtain

$$
(\alpha+\beta) x(0)=x_{0}-\alpha \int_{0}^{\tau} f(s, x(s)) d s-\beta \int_{0}^{\eta} f(s, x(s)) d s .
$$

Then

$$
x(0)=\frac{1}{\alpha+\beta}\left[x_{0}-\alpha \int_{0}^{\tau} f(s, x(s)) d s+\beta \int_{0}^{\eta} f(s, x(s)) d s\right]
$$

and

$$
x(t)=\frac{1}{\alpha+\beta}\left[x_{0}-\alpha \int_{0}^{\tau} f(s, x(s)) d s-\beta \int_{0}^{\eta} f(s, x(s)) d s\right]+\int_{0}^{t} f(s, x(s)) d s .
$$

Now, we have the following existence theorem

Theorem 1. Let the assumption (i)-(ii)-(iii) are satisfied, then there exists at least one absolutely continuous solution $x \in A C[0, T]$ of the Problem (1)-(2).

Proof. Define the operator $F$ by

$$
F x(t)=\frac{1}{\alpha+\beta}\left[x_{0}-\alpha \int_{0}^{\tau} f(s, x(s)) d s-\beta \int_{0}^{\eta} f(s, x(s)) d s\right]+\int_{0}^{t} f(s, x(s)) d s .
$$

Define the set

$$
Q_{r}=\{x:\|x\| \leq r\} \subset C[0, T], \quad r=\frac{\left|x_{0}\right|+2|| m||(\alpha+\beta)}{(\alpha+\beta)(1-2 b)} .
$$

Let $x \in Q_{r}$, then

$$
\begin{aligned}
|F x(t)| & =\left|\frac{1}{\alpha+\beta}\left[x_{0}-\alpha \int_{0}^{\tau} f(s, x(s)) d s-\beta \int_{0}^{\eta} f(s, x(s)) d s\right]+\int_{0}^{t} f(s, x(s)) d s\right| \\
& \leq \frac{1}{\alpha+\beta}\left[\left|x_{0}\right|+\alpha \int_{0}^{\tau}|f(s, x(s))| d s+\beta \int_{0}^{\eta}|f(s, x(s))| d s\right]+\int_{0}^{t}|f(s, x(s))| d s \\
& \leq \frac{1}{\alpha+\beta}\left[\left|x_{0}\right|+\alpha \int_{0}^{\tau}(|m(s)|+b|x|) d s+\beta \int_{0}^{\eta}(|m(s)|+b|x|) d s\right]+\int_{0}^{t}(|m(s)|+b|x|) d s
\end{aligned}
$$




$$
\begin{aligned}
& \leq \frac{1}{\alpha+\beta}\left[\left|x_{0}\right|+\alpha \int_{0}^{T}(|m(s)|+b|x|) d s+\beta \int_{0}^{T}(|m(s)|+b|x|) d s\right]+\int_{0}^{T}(|m(s)|+b|x|) d s \\
& \leq \frac{1}{\alpha+\beta}\left[\left|x_{0}\right|+\frac{\alpha}{\alpha+\beta}(\|m\|+b|| x|| T)+\frac{\beta}{\alpha+\beta}(|| m||+b|| x|| T)\right]+(\|m\|+b|| x|| T) \\
& \leq \frac{1}{\alpha+\beta}\left|x_{0}\right|+2(\|m\|+b\|x\| T) \leq r .
\end{aligned}
$$

Then the class of functions $\{F x\}$ is uniformly bounded on $Q_{r}$, and $F: Q_{r} \rightarrow Q_{r}$. Let $x \in Q_{r}$ and $t_{1}, t_{2} \in[0, T]$, such that $\left|t_{2}-t_{1}\right|<\delta$, then

$$
\begin{aligned}
\left|F x\left(t_{2}\right)-F x\left(t_{1}\right)\right|= & \mid \frac{1}{\alpha+\beta}\left[x_{0}-\alpha \int_{0}^{\tau} f(s, x(s)) d s-\beta \int_{0}^{\eta} f(s, x(s)) d s\right]+\int_{0}^{t_{2}} f(s, x(s)) d s \\
& -\frac{1}{\alpha+\beta}\left[x_{0}-\alpha \int_{0}^{\tau} f(s, x(s)) d s-\beta \int_{0}^{\eta} f(s, x(s)) d s\right]+\int_{0}^{t_{1}} f(s, x(s)) d s \mid \\
\leq & \int_{t_{1}}^{t_{2}}|f(s, x(s))| d s \\
\leq & \int_{t_{1}}^{t_{2}}(|m(s)|+b|x|) d s \\
\leq & \int_{t_{1}}^{t_{2}}|m(s)| d s+b|x|\left(t_{2}-t_{1}\right) .
\end{aligned}
$$

So, the class of functions $\{F x\}$ is equi-continuous on $Q_{r}$. From Arzela Theorem [18] we deduce that the class of functions $\{F x\}$ is compact, and $F: Q_{r} \rightarrow Q_{r}$ is compact. Now we prove that $F$ is continuous operator. For this let $\left\{x_{n}\right\} \subset Q_{r}$ be convergent sequence such that $x_{n}(t) \rightarrow x_{0}(t)$, then

$$
F x_{n}(t)=\frac{1}{\alpha+\beta}\left[x_{0}-\alpha \int_{0}^{\tau} f\left(s, x_{n}(s)\right) d s-\beta \int_{0}^{\eta} f\left(s, x_{n}(s)\right) d s\right]+\int_{0}^{t} f\left(s, x_{n}(s)\right) d s
$$

and

$$
\lim _{n \rightarrow \infty} F x_{n}(t)=\frac{1}{\alpha+\beta}\left[x_{0}-\alpha \lim _{n \rightarrow \infty} \int_{0}^{\tau} f\left(s, x_{n}(s)\right) d s-\beta \lim _{n \rightarrow \infty} \int_{0}^{\eta} f(s, x(s)) d s\right]+\lim _{n \rightarrow \infty} \int_{0}^{t} f\left(s, x_{n}(s)\right) d s .
$$

From assumptions (i), (ii), we have

$$
f\left(s, x_{n}(s)\right) \rightarrow f\left(s, x_{0}(s)\right)
$$

and

$$
|f(s, x(s))| \leq|m(s)|+b|x| \in L_{1}[0, T] .
$$

Applying Lebesgue dominated convergence Theorem [18], we have

$$
\begin{aligned}
\lim _{n \rightarrow \infty} F x_{n}(t) & =\frac{1}{\alpha+\beta}\left[x_{0}-\alpha \int_{0}^{\tau} \lim _{n \rightarrow \infty} f\left(s, x_{n}(s)\right) d s-\beta \int_{0}^{\eta} \lim _{n \rightarrow \infty} f\left(s, x_{n}(s)\right) d s\right]+\int_{0}^{t} \lim _{n \rightarrow \infty} f\left(s, x_{n}(s)\right) d s \\
& =\frac{1}{\alpha+\beta}\left[x_{0}-\alpha \int_{0}^{\tau} f\left(s, \lim _{n \rightarrow \infty} x_{n}(s)\right) d s-\beta \int_{0}^{\eta} f\left(s, \lim _{n \rightarrow \infty} x_{n}(s)\right) d s\right]+\int_{0}^{t} f\left(s, \lim _{n \rightarrow \infty} x_{n}(s)\right) d s \\
& =\frac{1}{\alpha+\beta}\left[x_{0}-\alpha \int_{0}^{\tau} f\left(s, x_{0}(s)\right) d s-\beta \int_{0}^{\eta} f\left(s, x_{0}(s)\right) d s\right]+\int_{0}^{t} f\left(s, x_{0}(s)\right) d s=F x_{0}(t) .
\end{aligned}
$$

Hence, $F: Q_{r} \rightarrow Q_{r}$ is continuous. Now by Schauder fixed point Theorem [19] there exists at least one solution $x \in C[0, T]$ of the Problem (1)-(2). Let $x \in C[0, T]$ be a solution of the Problem (1)-(2). Differentiating the integral Equation (7), we obtain

$$
\begin{aligned}
\frac{d x}{d t} & =\frac{d}{d t}\left(\frac{1}{\alpha+\beta}\left[x_{0}-\alpha \int_{0}^{\tau} f(s, x(s)) d s-\beta \int_{0}^{\eta} f(s, x(s)) d s\right]+\int_{0}^{t} f(s, x(s)) d s\right) \\
& =\frac{d}{d t} \int_{0}^{t} f(s, x(s)) d s .
\end{aligned}
$$


Since $f$ is measurable in $t \in[0, T]$ and bounded by integrable function, then $f \in L^{1}[0, T]$, and

$$
\frac{d x}{d t}=f(t, x(t)) \quad \text { a.e, } \quad t \in(0, T]
$$

Putting $t=\tau$ in the integral Equation (7), we get

$$
x(\tau)=\frac{1}{\alpha+\beta}\left[x_{0}-\alpha \int_{0}^{\tau} f(s, x(s)) d s-\beta \int_{0}^{\eta} f(s, x(s)) d s\right]+\int_{0}^{\tau} f(s, x(s)) d s .
$$

and

$$
\begin{aligned}
\alpha x(\tau) & =\frac{\alpha}{\alpha+\beta}\left[x_{0}-\frac{\alpha_{2}}{\alpha+\beta} \int_{0}^{\tau} f(s, x(s)) d s-\frac{\alpha \beta}{\alpha+\beta} \int_{0}^{\eta} f(s, x(s)) d s\right]+\alpha \int_{0}^{\tau} f(s, x(s)) d s \\
& =\frac{\alpha}{\alpha+\beta} x_{0}+\frac{\alpha \beta}{\alpha+\beta} \int_{0}^{\tau} f(s, x(s)) d s-\frac{\alpha \beta}{\alpha+\beta} \int_{0}^{\eta} f(s, x(s)) d s .
\end{aligned}
$$

Also

$$
\beta x(\eta)=\frac{\beta}{\alpha+\beta} x_{0}-\frac{\alpha \beta}{\alpha+\beta} \int_{0}^{\tau} f(s, x(s)) d s+\frac{\alpha \beta}{\alpha+\beta} \int_{0}^{\eta} f(s, x(s)) d s .
$$

Then

$$
\alpha x(\tau)+\beta x(\eta)=x_{0}, \quad \tau \in[0, T), \eta \in(0, T] .
$$

\subsection{Maximal and minimal solution}

Let $u(t)$ be a solution of the integral Equation (7), then $u(t)$ is said to be a maximal solution of (7), if for every solution of (7) satisfies the inequality:

$$
x(t) \leq u(t), \quad t \in[0, T] .
$$

A minimal solution $v(t)$ can be defined by similar way by reversing the above inequality i.e.,

$$
x(t)>v(t), \quad t \in[0, T] .
$$

We will use the following lemma to prove the existence of the maximal and minimal solutions.

Lemma 2. Let the assumption of Theorem 1 are satisfied and $x(t)$ and $y(t)$ are two continuous functions on $[0, T]$ satisfying

$$
\begin{aligned}
& x(t) \leq \frac{1}{\alpha+\beta}\left[x_{0}-\alpha \int_{0}^{\tau} f(s, x(s)) d s-\beta \int_{0}^{\eta} f(s, x(s)) d s\right]+\int_{0}^{t} f(s, x(s)) d s, \\
& y(t) \geq \frac{1}{\alpha+\beta}\left[x_{0}-\alpha \int_{0}^{\tau} f(s, y(s)) d s-\beta \int_{0}^{\eta} f(s, y(s)) d s\right]+\int_{0}^{t} f(s, y(s)) d s,
\end{aligned}
$$

and one of them is strict. If $f$ is monotonic nondecreasing in $x$, then

$$
x(t)<y(t), \quad t \in[0, T] .
$$

Proof. Let the conclusion (8) is false, then there exist $t_{1}$ such that

$$
x\left(t_{1}\right)=y\left(t_{2}\right) \quad t_{1}>0,
$$

and

$$
x(t)<y(t), \quad 0<t<t_{1} .
$$


From the monotonicity of $f(t, x(t))$ in $x$, we have

$$
\begin{aligned}
x\left(t_{1}\right) & \leq \frac{1}{\alpha+\beta}\left[x_{0}-\alpha \int_{0}^{\tau} f(s, x(s)) d s-\beta \int_{0}^{\eta} f(s, x(s)) d s\right]+\int_{0}^{t_{1}} f(s, x(s)) d s, \quad t \in[0, T] \\
& <\frac{1}{\alpha+\beta}\left[x_{0}-\alpha \int_{0}^{\tau} f(s, y(s)) d s-\beta \int_{0}^{\eta} f(s, y(s)) d s\right]+\int_{0}^{t_{1}} f(s, y(s)) d s, \\
& <y\left(t_{1}\right) .
\end{aligned}
$$

This contradicts the fact that $x\left(t_{1}\right)=y\left(t_{1}\right)$, then

$$
x(t)<y(t), \quad t \in[0, T]
$$

For the existence of the maximal and minimal solutions we have the following theorem.

Theorem 2. Let the assumptions of Theorem 1 are satisfied. If $f(t, x(t))$ is monotonic nondecreasing in $x$ for each $t \in[0, T]$, then the Equation (7) (consequently the Problem (1)-(2)) has maximal and minimal solutions.

Proof. Firstly we shall prove the existence of the maximal solution of (7). Let $\epsilon>0$ be given then consider the integral equation

$$
x_{\epsilon}(t) \leq \frac{1}{\alpha+\beta}\left[x_{0}-\alpha \int_{0}^{\tau} f_{\epsilon}\left(s, x_{\epsilon}(s)\right) d s-\beta \int_{0}^{\eta} f_{\epsilon}\left(s, x_{\epsilon}(s)\right) d s\right]+\int_{0}^{t_{1}} f(s, x(s)) d s,
$$

where

$$
f_{\epsilon}\left(t, x_{\epsilon}(t)\right)=f\left(t, x_{\epsilon}(t)\right)+\epsilon
$$

It is clear that the Equation (9) has at least one solution $x_{\epsilon}(t) \in C[0, T]$. Now, let $\epsilon_{1}$ and $\epsilon_{2}$ be such that $0<\epsilon_{2}<\epsilon_{1}<\epsilon$, then

$$
\begin{aligned}
x_{\epsilon_{2}}(t) & =\frac{1}{\alpha+\beta}\left[x_{0}-\alpha \int_{0}^{\tau} f_{\epsilon_{2}}\left(s, x_{\epsilon_{2}}(s)\right) d s-\beta \int_{0}^{\eta} f_{\epsilon_{2}}\left(s, x_{\epsilon_{2}}(s)\right) d s\right]+\int_{0}^{t} f_{\epsilon_{2}}\left(s, x_{\epsilon_{2}}(s)\right) d s \\
& =\frac{1}{\alpha+\beta}\left[x_{0}-\alpha \int_{0}^{\tau}\left(f\left(s, x_{\epsilon_{2}}(s)\right)+\epsilon_{2}\right) d s-\beta \int_{0}^{\eta}\left(f\left(s, x_{\epsilon_{2}}(s)\right)+\epsilon_{2}\right) d s\right]+\int_{0}^{t}\left(f\left(s, x_{\epsilon_{2}}(s)\right)+\epsilon_{2}\right) d s
\end{aligned}
$$

Also

$$
\begin{aligned}
x_{\epsilon_{1}}(t) & =\frac{1}{\alpha+\beta}\left[x_{0}-\alpha \int_{0}^{\tau} f_{\epsilon_{1}}\left(s, x_{\epsilon_{1}}(s)\right) d s-\beta \int_{0}^{\eta} f_{\epsilon_{1}}\left(s, x_{\epsilon_{1}}(s)\right) d s\right]+\int_{0}^{t} f_{\epsilon_{1}}\left(s, x_{\epsilon_{1}}(s)\right) d s \\
& =\frac{1}{\alpha+\beta}\left[x_{0}-\alpha \int_{0}^{\tau}\left(f\left(s, x_{\epsilon_{1}}(s)\right)+\epsilon_{1}\right) d s-\beta \int_{0}^{\eta}\left(f\left(s, x_{\epsilon_{1}}(s)\right)+\epsilon_{1}\right) d s\right]+\int_{0}^{t}\left(f\left(s, x_{\epsilon_{1}}(s)\right)+\epsilon_{1}\right) d s \\
& >\frac{1}{\alpha+\beta}\left[x_{0}-\alpha \int_{0}^{\tau}\left(f\left(s, x_{\epsilon_{2}}(s)\right)+\epsilon_{2}\right) d s-\beta \int_{0}^{\eta}\left(f\left(s, x_{\epsilon_{2}}(s)\right)+\epsilon_{2}\right) d s\right]+\int_{0}^{t}\left(f\left(s, x_{\epsilon_{2}}(s)\right)+\epsilon_{2}\right) d s
\end{aligned}
$$

Applying Lemma 2, we obtain

$$
x_{\epsilon_{2}}<x_{\epsilon_{1}}, \quad t \in[0, T]
$$

As shown before the family of function $x_{\epsilon}(t)$ is equi-continuous and uniformly bounded. Then, by Arzela Theorem [18], there exist a decreasing sequence $\epsilon_{n}$ such that $\epsilon_{0} \rightarrow 0$ as $n \rightarrow \infty$, and $u(t)=\lim _{n \rightarrow \infty} x_{\epsilon_{n}}(t)$ exists uniformly in $[0, T]$ and denote his limit by $u(t)$. From the continuity of the functions $f_{\epsilon}\left(t, x_{\epsilon}(t)\right)$, we get $f_{\epsilon}\left(t, x_{\epsilon}(t)\right) \rightarrow f(t, x(t))$ as $n \rightarrow \infty$ and

$$
u(t)=\lim _{n \rightarrow \infty} x_{\epsilon_{n}}(t)=\frac{1}{\alpha+\beta}\left[x_{0}-\alpha \int_{0}^{\tau} f(s, x(s)) d s-\beta \int_{0}^{\eta} f(s, x(s)) d s\right]+\int_{0}^{t} f(s, x(s)) d s .
$$


Now we prove that $u(t)$ is the maximal solution of (7). To do this, let $x(t)$ be any solution of (7), then

$$
\frac{1}{\alpha+\beta}\left[x_{0}-\alpha \int_{0}^{\tau} f(s, x(s)) d s-\beta \int_{0}^{\eta} f(s, x(s)) d s\right]+\int_{0}^{t} f(s, x(s)) d s
$$

and

$$
\begin{aligned}
x_{\epsilon}(t) & =\frac{1}{\alpha+\beta}\left[x_{0}-\alpha \int_{0}^{\tau} f_{\epsilon}\left(s, x_{\epsilon}(s)\right) d s-\beta \int_{0}^{\eta} f_{\epsilon}\left(s, x_{\epsilon}(s)\right) d s\right]+\int_{0}^{t} f_{\epsilon}\left(s, x_{\epsilon}(s)\right) d s \\
& =\frac{1}{\alpha+\beta}\left[x_{0}-\alpha \int_{0}^{\tau}\left(f\left(s, x_{\epsilon}(s)+\epsilon\right)\right) d s-\beta \int_{0}^{\eta}\left(f\left(s, x_{\epsilon}(s)\right)+\epsilon\right) d s\right]+\int_{0}^{t}\left(f\left(s, x_{\epsilon}(s)\right)+\epsilon\right) d s \\
& >\frac{1}{\alpha+\beta}\left[x_{0}-\alpha \int_{0}^{\tau} f\left(s, x_{\epsilon}(s)\right) d s-\beta \int_{0}^{\eta} f\left(s, x_{\epsilon}(s)\right) d s\right]+\int_{0}^{t} f\left(s, x_{\epsilon}(s)\right) d s=x(t)
\end{aligned}
$$

Applying Lemma 2, we obtain

$$
x(t)<x_{\epsilon}(t) \quad t \in[0, T] .
$$

From the uniqueness of the maximal solution, it is clear that $x_{\epsilon}(t)$ tends to $u(t)$ uniformly in $[0, T]$ as $\epsilon \rightarrow 0$. By similar way as done above we can prove the existence of the minimal solution.

\subsection{Uniqueness of the solution}

Consider the problem (1)-(2) under the following assumptions

$\left(i^{*}\right) f:[0, T] \times R \rightarrow R$ is measurable in $t \in[0, T]$ for every $x \in R$ satisfied the Lipschitz condition

$$
|f(t, x)-f(t, y)| \leq L|x-y|, \quad \text { and } \quad|f(t, 0)|=m(t)
$$

Theorem 3. Let the assumptions $\left(i^{*}\right)$ be satisfied. If $2 L T<1$, then the solution of the nonlocal two-point boundary value Problem (1)-(2) is unique.

Proof. From assumption $\left(i^{*}\right)$ we get

$$
|f(t, x)|-|f(t, 0)| \leq|f(t, x)-f(t, 0)| \leq b|x|
$$

and

$$
|f(t, x)| \leq b|x|+|f(t, 0)|=m(t)+b|x|
$$

Then the assumptions (ii) is satisfied, so there exists at least one solution $x \in A C[0, T]$ of the Problem (1)-(2). Let $x$ and $y$ be two solutions of the Problem (1)-(2), then we have

$$
\begin{aligned}
|x(t)-y(t)|= & \mid \frac{1}{\alpha+\beta}\left[x_{0}-\alpha \int_{0}^{\tau} \int_{0}^{t} f(s, x(s)) d s-\beta \int_{0}^{\eta} f(s, x(s)) d s\right]+\int_{0}^{t} f(s, x(s)) d s \\
& -\frac{1}{\alpha+\beta}\left[x_{0}-\alpha \int_{0}^{\tau} \int_{0}^{t} f(s, y(s)) d s-\beta \int_{0}^{\eta} f(s, y(s)) d s\right]+\int_{0}^{t} f(s, y(s)) d s \mid \\
\leq & \frac{\alpha}{\alpha+\beta} \int_{0}^{\tau}|f(s, x(s))-f(s, y(s))| d s+\frac{\beta}{\alpha+\beta} \int_{0}^{\eta}|f(s, x(s))-f(s, y(s))| d s \\
& +\int_{0}^{t}|f(s, x(s))-f(s, y(s))| d s \\
\leq & \frac{\alpha}{\alpha+\beta} L \int_{0}^{\tau}|x(s)-y(s)| d s+\frac{\beta}{\alpha+\beta} L \int_{0}^{\eta}|x(s)-y(s)| d s+L \int_{0}^{t}|x(s)-y(s)| d s \\
\leq & \frac{\alpha}{\alpha+\beta} L \int_{0}^{T}|x(s)-y(s)| d s+\frac{\beta}{\alpha+\beta} L \int_{0}^{T}|x(s)-y(s)| d s+L \int_{0}^{T}|x(s)-y(s)| d s \\
\leq & \frac{\alpha}{\alpha+\beta} L T|| x-y\left\|+\frac{\beta}{\alpha+\beta} L T|| x-y\right\|+L T|| x-y|| .
\end{aligned}
$$


Then

$$
\|x-y\| \leq 2 L T|| x-y \|
$$

and

$$
\|x-y\|(1-2 L T) \leq 0 \Rightarrow\|x-y\|=0 \Rightarrow x=y .
$$

Hence, the solution of the integral Equation (7) (consequently the Problem (1)-(2)) is unique solution $x \in A C[0, T]$.

\section{Continuous dependence of the solution}

Definition 1. The solution of the nonlocal two-point boundary value Problem (1)-(2) depends continuously on $x_{0}$, if $\forall \epsilon>0, \exists \delta>0$, we have

$$
\left|x-x_{0}^{*}\right| \leq \delta \Rightarrow\left\|x-x^{*}\right\| \leq \epsilon .
$$

where $x^{*} \in A C[0, T]$ is the unique solution of the nonlocal two-point boundary value Problem (1)-(2).

Theorem 4. Let the assumption of Theorem 3 are satisfied, then the solution of a nonlocal two-points boundary value Problem (1)-(2) is dependence continuously on $x_{0}$.

Proof. let $x, x^{*}$ be the solutions of a nonlocal two-points boundary value Problem (1)-(2), then

$$
\begin{aligned}
\left|x(t)-x^{*}(t)\right|= & \mid \frac{1}{\alpha+\beta}\left[x_{0}-\beta \int_{0}^{\tau} f(s, x(s)) d s-\beta \int_{0}^{\eta} f(s, x(s)) d s\right]+\int_{0}^{t} f(s, x(s)) d s \\
& -\frac{1}{\alpha+\beta}\left[x_{0}^{*}-\beta \int_{0}^{\tau} f\left(s, x^{*}(s)\right) d s-\beta \int_{0}^{\eta} f\left(s, x^{*}(s)\right) d s\right]+\int_{0}^{t} f\left(s, x^{*}(s)\right) d s \mid \\
\leq & \frac{1}{\alpha+\beta}\left|x_{0}-x_{0}^{*}\right|+\frac{\alpha}{\alpha+\beta} \int_{0}^{\tau}\left|f(s, x(s))-f\left(s, x^{*}(s)\right)\right| d s \\
& +\frac{\beta}{\alpha+\beta} \int_{0}^{\eta}\left|f(s, x(s))-f\left(s, x^{*}(s)\right)\right| d s+\int_{0}^{t}\left|f(s, x(s))-f\left(s, x^{*}(s)\right)\right| d s \\
\leq & \frac{1}{\alpha+\beta}\left|x_{0}-x_{0}^{*}\right|+\frac{\alpha}{\alpha+\beta} L \int_{0}^{T}\left|x(s)-x^{*}(s)\right| d s \\
& +\frac{\beta}{\alpha+\beta} L \int_{0}^{T}\left|x(s)-x^{*}(s)\right| d s+L \int_{0}^{T}\left|x(s)-x^{*}(s)\right| d s \\
\leq & \frac{\delta}{\alpha+\beta}+2 L T|| x-x^{*}|| .
\end{aligned}
$$

Then

$$
\left\|x-x^{*}\right\|(1-2 L T) \leq \frac{\delta}{\alpha+\beta} \text { implies } \quad\left\|x-x^{*}\right\| \leq \frac{\delta}{(1-2 L T)(\alpha+\beta}=\epsilon .
$$

This prove the continuous dependence of solution of the nonlocal two-point boundary value Problem (1)-(2) on $x_{0}$.

Definition 2. The solution of the nonlocal two-point boundary value Problem (1)-(2) depends continuously on $\alpha$ and $\beta$, if $\forall \epsilon>0, \exists \delta>0$, we have

$$
\left|\alpha-\alpha^{*}\right| \leq \delta_{1}, \quad\left|\beta-\beta^{*}\right| \leq \delta_{2} \Rightarrow\left\|x-x^{*}\right\| \leq \epsilon,
$$

where $x^{*}$ is the unique solution of the nonlocal two-points boundary value Problem (1)-(2).

Theorem 5. Let the assumption of Theorem 3 is satisfied, then the solution of the nonlocal two-point boundary value Problem (1)-(2) is depends continuously on $\alpha, \beta$. 
Proof. let $x, x^{*}$ be the solutions of the nonlocal two-points boundary value Problem (1)-(2), then

$$
\begin{aligned}
\left|x(t)-x^{*}(t)\right|= & \mid \frac{x_{0}}{\alpha+\beta}-\frac{\alpha}{\alpha+\beta} \int_{0}^{\tau} f(s, x(s)) d s-\frac{\beta}{\alpha+\beta} \int_{0}^{\eta} f(s, x(s)) d s \\
& +\int_{0}^{t} f(s, x(s)) d s-\frac{1}{\alpha^{*}+\beta^{*}} x_{0}+\frac{\alpha^{*}}{\alpha^{*}+\beta^{*}} \int_{0}^{\tau} f\left(s, x^{*}(s)\right) d s \\
& +\frac{\beta^{*}}{\alpha^{*}+\beta^{*}} \int_{0}^{\eta} f\left(s, x^{*}(s)\right) d s-\int_{0}^{t} f\left(s, x^{*}(s)\right) d s \mid \\
= & \mid \frac{\left(\alpha^{*}-\alpha\right)\left(\beta^{*}-\beta\right)}{(\alpha+\beta)\left(\alpha^{*}+\beta\right)} x_{0}-\frac{\alpha}{\alpha+\beta} \int_{0}^{\tau} f(s, x(s)) d s+\frac{\alpha}{\alpha+\beta} \int_{0}^{\tau} f\left(s, x^{*}(s)\right) d s \\
& -\frac{\alpha}{\alpha+\beta} \int_{0}^{\tau} f\left(s, x^{*}(s)\right) d s-\frac{\beta}{\alpha+\beta} \int_{0}^{\eta} f(s, x(s)) d s-\frac{\beta}{\alpha+\beta} \int_{0}^{\eta} f\left(s, x^{*}(s)\right) d s \\
& -\frac{\beta}{\alpha^{*}+\beta} \int_{0}^{\eta} f\left(s, x^{*}(s)\right) d s+\frac{\beta}{\alpha^{*}+\beta} \int_{0}^{\tau} f\left(s, x^{*}(s)\right) d s \\
& +\frac{\beta^{*}}{\alpha^{*}+\beta} \int_{0}^{\eta} f\left(s, x^{*}(s)\right) d s+\int_{0}^{t}\left(f(s, x(s))-f\left(s, x^{*}(s)\right)\right) d s \mid \\
\leq & \frac{\left(\alpha^{*}-\alpha\right)\left(\beta^{*}-\beta\right)}{(\alpha+\beta)\left(\alpha^{*}+\beta\right)}\left|x_{0}\right|+\frac{\alpha}{\alpha+\beta} \int_{0}^{\tau}\left|f\left(s, x^{*}(s)\right)-f(s, x(s))\right| d s \\
& +\frac{\beta}{\alpha+\beta} \int_{0}^{\eta}\left|f(s, x(s))-f\left(s, x^{*}(s)\right)\right| d s+\frac{\alpha^{*}(\alpha+\beta)-\alpha\left(\alpha^{*}+\beta^{*}\right)}{(\alpha+\beta)\left(\alpha^{*}+\beta\right)} \int_{0}^{\tau}\left|f\left(s, x^{*}(s)\right)\right| d s \\
& +\frac{\beta^{*}(\alpha+\beta)-\beta\left(\alpha^{*}+\beta^{*}\right)}{(\alpha+\beta)\left(\alpha^{*}+\beta\right)} \int_{0}^{\eta}\left|f\left(s, x^{*}(s)\right)\right| d s+\int_{0}^{t}\left(f(s, x(s))-f\left(s, x^{*}(s)\right)\right) d s \mid \\
\leq & \frac{\delta_{1}+\delta_{2}}{(\alpha+\beta)\left(\alpha^{*}+\beta\right)}\left|x_{0}\right|+\frac{\alpha}{\alpha+\beta} L \int_{0}^{T}\left|x(s)-x^{*}(s)\right| d s \\
& +\frac{\beta}{\alpha+\beta} L \int_{0}^{T}\left|x(s)-x^{*}(s)\right| d s+\frac{\alpha^{*}(\alpha+\beta)-\alpha\left(\alpha^{*}+\beta^{*}\right)}{(\alpha+\beta)\left(\alpha^{*}+\beta\right)} \int_{0}^{T}\left|f\left(s, x^{*}(s)\right)\right| d s \\
& +\frac{\beta^{*}(\alpha+\beta)-\beta\left(\alpha^{*}+\beta^{*}\right)}{(\alpha+\beta)\left(\alpha^{*}+\beta\right)} \int_{0}^{T}\left|f\left(s, x^{*}(s)\right)\right| d s+L \int_{0}^{T}\left|x(s)-x^{*}(s)\right| d s . \\
&
\end{aligned}
$$

Then

$$
\begin{aligned}
\left\|x-x^{*}\right\| \leq & \frac{\left(\delta_{1}+\delta_{2}\right.}{(\alpha+\beta)\left(\alpha^{*}+\beta^{*}\right)}\left|x_{0}\right|+\frac{\alpha}{\alpha+\beta} L T\left\|x-x^{*}\right\|+\frac{\beta}{\alpha+\beta} L T\left\|x-x^{*}\right\|+\frac{\alpha^{*} \beta-\alpha \beta^{*}}{(\alpha+\beta)\left(\alpha^{*}+\beta^{*}\right)} M \\
& +\frac{\beta^{*} \alpha-\beta \alpha^{*}}{(\alpha+\beta)\left(\alpha^{*}+\beta^{*}\right)} M+L T\left\|x-x^{*}\right\| \\
\leq & \frac{\delta_{1}+\delta_{2}}{(\alpha+\beta)\left(\alpha^{*}+\beta^{*}\right)}\left|x_{0}\right|+2 L T\left\|x-x^{*}\right\|
\end{aligned}
$$

and

$$
(1-2 L T)|| x-x^{*}|| \leq \frac{\delta_{1}+\delta_{2}}{(\alpha+\beta)\left(\alpha^{*}+\beta^{*}\right)}\left|x_{0}\right| \text { implies } \quad|| x-x^{*}|| \leq \frac{\mid \delta_{1}+\delta_{2}}{(\alpha+\beta)\left(\alpha^{*}+\beta^{*}\right)(1-2 L T)}\left|x_{0}\right|=\epsilon .
$$

This prove that $\forall \epsilon>0, \quad \exists \delta(\epsilon)>0$ such that

$$
\left|\alpha-\alpha^{*}\right| \leq \delta_{1},\left|\beta-\beta^{*}\right| \leq \delta_{2} \Rightarrow|| x-x^{*}|| \leq \epsilon .
$$

\section{Anti-periodic boundary value problem}

Consider the nonlocal boundary value problem of the differential equation (1) with the anti-periodic nonlocal condition

$$
x(\tau)=-x(1-\tau), \quad \tau \in[0, T]
$$

we have the following corollary; 
Corollary 6. If $\alpha=1, \beta=1$ and $\eta=1-\tau$ and $x_{0}=0$ in Theorem 1 , then the anti-periodic boundary value problem

$$
\begin{aligned}
\frac{d x}{d t} & =f(t, x(t)), \quad t \in(0, T) \\
x(\tau) & =-x(1-\tau), \quad \tau \in(0, T)
\end{aligned}
$$

has the at lease one solution $x \in A C[0, T]$

$$
x(t)=-\int_{0}^{\tau} f(s, x(s)) d s-\int_{0}^{1-\tau} f(s, x(s)) d s+\int_{0}^{t} f(s, x(s)) d s .
$$

Now, let $\tau=\frac{1}{2}$, then

$$
\begin{aligned}
x(t) & =-\int_{0}^{\frac{1}{2}} f(s, x(s)) d s-\int_{0}^{\frac{1}{2}} f(s, x(s)) d s+\int_{0}^{t} f(s, x(s)) d s \\
& =\int_{0}^{t} f(s, x(s)) d s-2 \int_{0}^{\frac{1}{2}} f(s, x(s)) d s .
\end{aligned}
$$

\section{Examples}

Example 1. Consider the differential Equation (1) with the backward condition $x(T)=x_{0}$, we have the following corollary;

Corollary 7. Let $\alpha=1, \beta=0$ and $\eta=T$ in Theorem 1, then the backward problem

$$
\begin{gathered}
\frac{d x}{d t}=f(t, x(t)) \\
x(T)=x_{0}
\end{gathered}
$$

has the solution $x \in A C[0, T]$

$$
\begin{aligned}
x(t) & =x_{0}-\int_{0}^{T} f(s, x(s)) d s+\int_{0}^{t} f(s, x(s)) d s \\
& =x_{0}-\int_{t}^{T} f(s, x(s)) d s .
\end{aligned}
$$

Example 2. Consider the differential Equation (1) with the forward condition $x(0)=x_{0}$, we have the following corollary;

Corollary 8. Let $\alpha=0, \beta=1$ and $\tau=0$ in Theorem 1 , then the initial value problem

$$
\begin{gathered}
\frac{d x}{d t}=f(t, x(t)) \\
x(0)=x_{0}
\end{gathered}
$$

has the solution $x \in A C[0, T]$

$$
x(t)=x_{0}+\int_{0}^{t} f(s, x(s)) d s
$$

\section{Conclusions}

We proved here, under certain conditions, the existence of at least one absolutely continuous solution $x \in A C[0, T]$ of the nonlocal two-point, with parameters $\left(\alpha, \beta\right.$ and $\left.x_{0}\right)$ boundary value Problem (1)-(2). The maximal and minimal solutions of the Problem (1)-(2) have been proved. The continuous dependence of the unique solution on the parameters $\alpha, \beta$ and $x_{0}$ ) have been also proved. The anti-periodic boundary value problem have been considered as an application. 
Author Contributions: All authors contributed equally to the writing of this paper. All authors read and approved the final manuscript.

Conflicts of Interest: "The authors declare no conflict of interest."

\section{References}

[1] Ladde, G. S., Lakshmikantham, V., \& Vatsala, A. S. (1985). Monotone iterative techniques for nonlinear differential equations (Vol. 27). Pitman Publishing.

[2] Lakshmikantham, V., \& Leela, S. (1983). Existence and monotone method for periodic solutions of first-order differential equations. Journal of Mathematical Analysis and Applications, 91(1), 237-243.

[3] Liu, H., \& Jiang, D. (2000). Two-point boundary value problem for first order implicit differential equations. Hiroshima Mathematical Journal, 30(1), 21-27.

[4] Ahmad, B., \& Nieto, J. J. (2009). Existence results for a coupled system of nonlinear fractional differential equations with three-point boundary conditions. Computers \& Mathematics with Applications, 58(9), 1838-1843.

[5] Boucherif, A., \& Precup, R. (2003). On the nonlocal initial value problem for first order differential equations. Fixed Point Theory, 4(2), 205-212.

[6] Byszewski, L. (1991). Theorems about the existence and uniqueness of solutions of a semilinear evolution nonlocal Cauchy problem. Journal of Mathematical analysis and Applications, 162(2), 494-505.

[7] Dong, X., Wang, J., \& Zhou, Y. (2011). On nonlocal problems for fractional differential equations in Banach spaces. Opuscula Mathematica, 31(3), 341-357.

[8] El-Sayed, A. M. A., \& Bin-Taher, E. O. (2011). A nonlocal problem for a multiterm fractional-order differential equation. International Journal of Mathematical Analysis, 5(29), 1445-1451.

[9] El-Sayed, A. M. A., Hamdallah, E. M., \& Elkadeky, K. W. (2012). Solution of a class of nonlocal problem for the differential inclusion $x^{\prime}(t)=F(t, x(t))$ Egy. Chin.J Comp. App. Math, 1(1), 24-30.

[10] El-Sayed, A. M. A., Hamdallah, E. M., \& Elkadeky, K. W. (2012). Uniformly stable positive monotonic solution of a nonlocal Cauchy problem. Advances in pure Mathematics, 2(2), 109-113.

[11] El-Sayed, A. M. A., Abd El-Rahman, R. O., \& El-Gendy, M. (2013). Uniformly stable solution of a nonlocal problem of coupled system of differential equations. Differential Equattions and applications, 5(3), 355-365.

[12] El-Sayed, A., \& Gamal, R. (2019). Infinite point and RiemannÜStieltjes integral conditions for an integro-differential equation. Nonlinear Analysis: Modelling and Control, 24(5), 733-754.

[13] El-Sayed, A. M. A., \& Ahmed, R. G. (2019). Existence of Solutions for a Functional Integro-Differential Equation with Infinite Point and Integral Conditions. International Journal of Applied and Computational Mathematics, 5(4), 108.

[14] El-Sayed, A. M. A., \& Ahmed, R. G. (2020). Solvability of a coupled system of functional integro-differential equations with infinite point and RiemannŰStieltjes integral conditions. Applied Mathematics and Computation, 370, 124918.

[15] Elkadeky, K. W. (2012). A nonlocal problem of the differential equation $x^{\prime}(t)=F\left(t, x, x^{\prime}\right)$. Journal of fractional Calculus and Applications,3(S), 1-8.

[16] Karakostas, G. L., \& Tsamatos, P. C. (2002). Existence of multiple positive solutions for a nonlocal boundary value problem. Topological Methods in Nonlinear Analysis, 19(1), 109-121.

[17] Xue, X. (2005). Nonlinear differential equations with nonlocal conditions in Banach spaces. Nonlinear Analysis: Theory, Methods E Applications, 63(4), 575-586.

[18] Goebel, K., \& Kirk, W. A. (1990). Topics in metric fixed point theory (Vol. 28). Cambridge University Press.

[19] Kolmogorov, A. N., \& Fomin, S. V. (1970). Introductory Real Analysis (Silverman, translator and ed.), RA Dover Publ. Inc., New York.

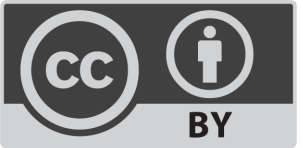

(C) 2020 by the authors; licensee PSRP, Lahore, Pakistan. This article is an open access article distributed under the terms and conditions of the Creative Commons Attribution (CC-BY) license (http://creativecommons.org/licenses/by/4.0/). 